\title{
An analogue of the Sommerfeld radiation condition for the Dirac operator.
}

\author{
Vladislav V. Kravchenko, Raúl Castillo P. \\ Departamento de Telecomunicaciones, \\ Escuela Superior de Ingeniería Mecánica y Eléctrica, \\ Instituto Politécnico Nacional, C.P. 07738, D.F., MEXICO \\ e-mail: vkravche@maya.esimez.ipn.mx \\ raulcp@tesla.esimez.ipn.mx
}

\begin{abstract}
A simple radiation condition at infinity for time-harmonic massive Dirac spinors is proposed. This condition allows an analogue of the Cauchy integral formula in unbounded domains for null-solutions of the Dirac equation to be proved. The result is obtained with the aid of methods of quaternionic analysis.
\end{abstract}

Key words: Dirac operator, radiation condition.

AMS subject classification: 30G35, 81Q05.

\section{Introduction}

There are two principal ways to guarantee the unicity of solution of a not homogeneous differential equation of mathematical physics in the whole space. The first is the famous limiting absorption principle which in the case of some operators, for example the Helmholtz operator, has a very natural physical meaning reflected in its name (see, e.g., [12]). The medium is assumed to be slightly absorbent with a corresponding absorption parameter $\varepsilon$. For $\varepsilon>0$ the inhomogeneous equation (under some additional natural conditions) has a unique solution $u_{\varepsilon}$ decreasing at infinity, and the solution for $\varepsilon=0$ is obtained as the limit of $u_{\varepsilon}$ when $\varepsilon \rightarrow 0$. This approach, based on a deep understanding of the physical nature of the differential operator, is not applicable in all cases. Another approach is to impose a radiation condition at infinity which "prohibits" the existence of some unwanted solutions. Such a prohibition is related to the unicity of an integral representation for null-solutions of the differential operator. In the case of the Helmholtz operator the corresponding radiation condition was introduced by A. Sommerfeld [10]. 
For the Maxwell equations such condition was proposed by C. Müller [8] and S. Silver [9]. Recently, A. McIntosh and M. Mitrea in [7] introduced a radiation condition for a special perturbed Clifford-Dirac operator. We use their approach to obtain a radiation condition for the operator $D+M^{\vec{\alpha}}$, where $D$ is the MoisilTheodoresco operator and $M^{\vec{\alpha}}$ is the operator of multiplication by a complex quaternion $\vec{\alpha}$ from the right-hand side (for exact definitions see Section 2). Then using a simple matrix transformation proposed in [4], which converts the operator $D+M^{\vec{\alpha}}$ into the Dirac operator in its traditional form through the $\gamma$-matrices, we rewrite the radiation condition for the Dirac spinors in a form suitable for applications and obtain an integral representation for null-solutions of the classical Dirac operator in unbounded domains, based on the proposed radiation condition.

\section{Preliminaries}

\subsection{Quaternions}

A quaternion $a$ can be represented as a linear combination of its components $\left(\left\{a_{k}\right\} \subset \mathbb{R}\right.$ for real quaternions and $\left\{a_{k}\right\} \subset \mathbb{C}$ for complex quaternions) and the elements of the orthonormal basis $i_{k}$ as follows

$$
a=\sum_{k=0}^{3} a_{k} i_{k}
$$

where $i_{0}:=1$ and $\left\{i_{k} \mid k=1,2,3\right\}$ are the quaternionic imaginary units, which have the following properties:

$$
\begin{gathered}
i_{k}^{2}=-1, \quad k=1,2,3 \\
i_{1} i_{2}=-i_{2} i_{1}=i_{3} ; \quad i_{2} i_{3}=-i_{3} i_{2}=i_{1} ; \quad i_{3} i_{1}=-i_{1} i_{3}=i_{2} .
\end{gathered}
$$

The imaginary unit in $\mathbb{C}$ is denoted as usual by $i$ and by definition commutes with quaternionic imaginary units.

$$
i \cdot i_{k}=i_{k} \cdot i, \quad k=\overline{0,3} .
$$

In some cases it is useful to represent a quaternion $a$ as the sum of a scalar part $\operatorname{Sc}(a)=a_{0}$ and a vectorial part $\operatorname{Vec}(a)=\vec{a}=a_{1} i_{1}+a_{2} i_{2}+a_{3} i_{3}$. Using this representation we can define the product of two arbitrary quaternions $a$ and $b$ in the form

$$
a \cdot b=a_{0} b_{0}-\langle\vec{a}, \vec{b}\rangle+a_{0} \vec{b}+\vec{a} b_{0}+[\vec{a} \times \vec{b}],
$$

where $\langle\cdot, \cdot\rangle$ is the scalar product and $[\cdot \times \cdot]$ is the vector product. 
The quaternionic conjugation of a quaternion $a=a_{0}+\vec{a}$ is defined as $\bar{a}=a_{0}-\vec{a}$. From (2) it is easy to observe that

$$
a \cdot \bar{a}=a_{0}^{2}+a_{1}^{2}+a_{2}^{2}+a_{3}^{2}=:|a|^{2},
$$

and to obtain an important property of quaternionic conjugation:

$$
\overline{a \cdot b}=\bar{b} \cdot \bar{a}
$$

Note that $|a \cdot b|=|a| \cdot|b|$.

One important conclusion which can be made using (3) is that each non-zero real quaternion $a$ is invertible and its inverse is given by

$$
a^{-1}=\frac{\bar{a}}{|a|^{2}} \text {. }
$$

We denote the set of zero divisors in $\mathbb{H}(\mathbb{C})$ by $\mathfrak{S}$ :

$$
\mathfrak{S}:=\{a \in \mathbb{H}(\mathbb{C}) \mid a \neq 0 ; \exists b \neq 0: a \cdot b=0\} .
$$

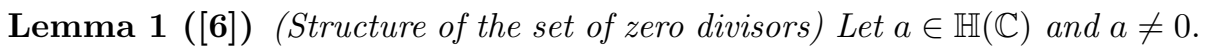
The following statements are equivalent:

1. $a \in \mathfrak{S}$.

2. $a \cdot \bar{a}=0$.

3. $a_{0}^{2}=\vec{a}^{2}$.

4. $a^{2}=2 a_{0} a=2 \vec{a} a$.

As the modulus introduced by (3) does not in general give information about the absolute values of the components of a complex quaternion when it is a zero divisor, another kind of modulus is frequently used

$$
|a|_{c}:=\sqrt{\left|a_{0}\right|^{2}+\left|a_{1}\right|^{2}+\left|a_{2}\right|^{2}+\left|a_{3}\right|^{2}}
$$

where $\left|a_{k}\right|^{2}=a_{k} a_{k}^{*}$ and "*" stands for the usual complex conjugation. (4) can be expressed also in the following ways

$$
|a|_{c}^{2}=|\operatorname{Re} a|^{2}+|\operatorname{Im} a|^{2}=\operatorname{Sc}\left(a \cdot \bar{a}^{*}\right)=\operatorname{Sc}\left(\bar{a}^{*} \cdot a\right) .
$$




\subsection{Quaternionic differential operators}

Let $\Omega \subset \mathbb{R}^{3}$ and $f \in C^{1}(\Omega ; \mathbb{H}(\mathbb{C}))$. The Moisil-Theodoresco operator is defined as

$$
D f:=\sum_{k=1}^{3} i_{k} \partial_{k} f
$$

where $\partial_{k}:=\frac{\partial}{\partial x_{k}}$. An important property of $D$ is obtained from (B)

$$
D^{2}=-\Delta,
$$

where $\Delta=\partial_{1}^{2}+\partial_{2}^{2}+\partial_{3}^{2}$ is the usual Laplace operator.

We will consider the following operator

$$
D_{ \pm \nu}:=D \pm \nu I
$$

where $\nu$ is a complex number, which was studied for the first time in [2] (for $\nu$ real). With the aid of the operator $D_{\nu}$ it is possible to factorize the Helmholtz operator

$$
\Delta+\nu^{2}=-(D+\nu)(D-\nu)=-D_{\nu} D_{-\nu} .
$$

Suppose that $\theta_{\nu}$ is a fundamental solution of the Helmholtz operator

$$
\left(\Delta+\nu^{2}\right) \theta_{\nu}=\delta
$$

Then, using (6) we obtain the functions

$$
\mathcal{K}_{ \pm \nu}:=-(D \mp \nu) \theta_{\nu}
$$

which are the fundamental solutions of $D_{ \pm \nu}$, that is

$$
D_{ \pm \nu} \mathcal{K}_{ \pm \nu}=\delta
$$

Let us assume that $\operatorname{Im} \nu \geq 0$. Under this condition, the fundamental solution of the Helmholtz operator is chosen as

$$
\theta_{\nu}(x)=-\frac{e^{i \nu|x|}}{4 \pi|x|}
$$

in which case it fulfills the Sommerfeld radiation condition at infinity and has a clear physical sense of an outgoing wave generated by a point source at the origin.

Substituting (8) into equality (7), we obtain

$$
\mathcal{K}_{ \pm \nu}(x)=-D \theta_{\nu}(x) \pm \nu \theta_{\nu}(x)=\left( \pm \nu+\frac{x}{|x|^{2}}-i \nu \frac{x}{|x|}\right) \theta_{\nu}(x)
$$


where $x:=\sum_{k=1}^{3} x_{k} i_{k}$. These functions were obtained in [5], (see also [6, Section $3])$.

Let us assume that $\Omega$ is a bounded domain in $\mathbb{R}^{3}$ with a piecewise smooth boundary $\Gamma:=\partial \Omega$.

Theorem 2 (Quaternionic Stokes' formula) Let $f$ and $g$ belong to $C^{1}(\Omega ; \mathbb{H}(\mathbb{C})) \cap$ $C(\bar{\Omega} ; \mathbb{H}(\mathbb{C}))$. Then

$$
\int_{\Omega}\left(\left(D_{r} f(y)\right) g(y)+f(y)(D g(y))\right) d y=\int_{\Gamma} f(y) \vec{n}(y) g(y) d \Gamma_{y},
$$

where $\vec{n}(y)$ is the quaternionic representation of the outward unit normal to the surface $\Gamma: \vec{n}(y)=\sum_{k=1}^{3} i_{k} n_{k}(y)$ and $D_{r} f:=\sum_{k=1}^{3} \partial_{k} f i_{k}$ is the right Moisil-Theodoresco operator.

The proof of this fact can be found in [6, Chapter 4] or [3, p. 86].

Let us consider the following integral operator

$$
K_{\nu}[f](x):=-\int_{\Gamma} \mathcal{K}_{\nu}(x-y) \vec{n}(y) f(y) d \Gamma_{y}, \quad x \in \mathbb{R}^{3} \backslash \Gamma
$$

which can be considered as an analogue of the Cauchy integral operator from complex analysis due to the following fact.

Theorem 3 (Quaternionic Cauchy integral formula) Let $f \in C^{1}(\Omega ; \mathbb{H}(\mathbb{C})) \cap$ $C(\bar{\Omega} ; \mathbb{H}(\mathbb{C}))$ and $f \in \operatorname{ker} D_{\nu}(\Omega)$. Then

$$
f(x)=K_{\nu}[f](x), \quad \forall x \in \Omega .
$$

\subsection{Dirac operator in quaternionic form}

Denote $\widetilde{\Phi}:=\Phi\left(t, x_{1}, x_{2},-x_{3}\right)$. The domain $\widetilde{\mathcal{G}}$ is assumed to be obtained from the domain $\mathcal{G} \subset \mathbb{R}^{4}$ by the reflection $x_{3} \rightarrow-x_{3}$. Let us consider the transformation denoted by $A$ and introduced in the following way. A function $\Phi: \mathcal{G} \subset \mathbb{R}^{4} \rightarrow \mathbb{C}^{4}$ is transformed into a function $\rho: \widetilde{\mathcal{G}} \subset \mathbb{R}^{4} \rightarrow \mathbb{H}(\mathbb{C})$ by the rule

$$
\rho=A[\Phi]:=\frac{1}{2}\left(-\left(\widetilde{\Phi_{1}}-\widetilde{\Phi_{2}}\right) i_{0}+i\left(\widetilde{\Phi_{0}}-\widetilde{\Phi_{3}}\right) i_{1}-\left(\widetilde{\Phi_{0}}+\widetilde{\Phi_{3}}\right) i_{2}+i\left(\widetilde{\Phi_{1}}+\widetilde{\Phi_{2}}\right) i_{3}\right) .
$$

Then the definition of the inverse transformation $A^{-1}$ is

$$
A^{-1}[\rho]=\left(-i \widetilde{\rho}_{1}-\widetilde{\rho}_{2},-\widetilde{\rho}_{0}-i \widetilde{\rho}_{3}, \widetilde{\rho}_{0}-i \widetilde{\rho}_{3}, i \widetilde{\rho}_{1}-\widetilde{\rho}_{2}\right)
$$


These transformations can be represented in a more explicit matrix form:

$$
\rho=A[\Phi]=\frac{1}{2}\left(\begin{array}{rrrr}
0 & -1 & 1 & 0 \\
i & 0 & 0 & -i \\
-1 & 0 & 0 & -1 \\
0 & i & i & 0
\end{array}\right)\left(\begin{array}{c}
\widetilde{\Phi_{0}} \\
\widetilde{\Phi_{1}} \\
\widetilde{\Phi_{2}} \\
\widetilde{\Phi_{3}}
\end{array}\right)
$$

and

$$
\Phi=A^{-1}[\rho]=\left(\begin{array}{rrrr}
0 & -i & -1 & 0 \\
-1 & 0 & 0 & -i \\
1 & 0 & 0 & -i \\
0 & i & -1 & 0
\end{array}\right)\left(\begin{array}{c}
\widetilde{\rho}_{0} \\
\widetilde{\rho}_{1} \\
\widetilde{\rho}_{2} \\
\widetilde{\rho}_{3}
\end{array}\right)
$$

The Dirac $\gamma$-matrices in the standard ([1], [1]) Dirac-Pauli form are

$$
\begin{aligned}
\gamma_{0}:= & \left(\begin{array}{rrrr}
1 & 0 & 0 & 0 \\
0 & 1 & 0 & 0 \\
0 & 0 & -1 & 0 \\
0 & 0 & 0 & -1
\end{array}\right), \quad \gamma_{1}:=\left(\begin{array}{rrrr}
0 & 0 & 0 & -1 \\
0 & 0 & -1 & 0 \\
0 & 1 & 0 & 0 \\
1 & 0 & 0 & 0
\end{array}\right), \\
\gamma_{2}:= & \left.\gamma_{3}:=\left(\begin{array}{rrrr}
0 & 0 & 0 & i \\
0 & 0 & -i & 0 \\
0 & -i & 0 & 0 \\
i & 0 & 0 & 0
\end{array}\right), \quad \begin{array}{rrrr}
0 & 0 & -1 & 0 \\
0 & 0 & 0 & 1 \\
1 & 0 & 0 & 0 \\
0 & -1 & 0 & 0
\end{array}\right) ; \\
\gamma_{5}:=i \gamma_{0} \gamma_{1} \gamma_{2} \gamma_{3} & =\left(\begin{array}{rrrr}
0 & 0 & -1 & 0 \\
0 & 0 & 0 & -1 \\
-1 & 0 & 0 & 0 \\
0 & -1 & 0 & 0
\end{array}\right) .
\end{aligned}
$$

Recall that:

1. $\gamma_{0}^{2}=\gamma_{5}^{2}=E_{4}$, the identity matrix;

2. $\gamma_{k}^{2}=-E_{4}, k=1,2,3$;

3. $\gamma_{j} \gamma_{k}=-\gamma_{k} \gamma_{j}, j, k=0,1,2,3,5, j \neq k$.

The transforms $A$ and $A^{-1}$ have the following algebraic properties [4]:

1. $A \gamma_{1} \gamma_{2} \gamma_{3} \gamma_{1} A^{-1}[\rho]=i_{1} \rho$

2. $A \gamma_{1} \gamma_{2} \gamma_{3} \gamma_{2} A^{-1}[\rho]=i_{2} \rho$

3. $A \gamma_{1} \gamma_{2} \gamma_{3} \gamma_{3} A^{-1}[\rho]=-i_{3} \rho$

4. $A \gamma_{1} \gamma_{2} \gamma_{3} \gamma_{0} A^{-1}[\rho]=\rho i_{1}$

5. $A \gamma_{1} \gamma_{2} \gamma_{3} A^{-1}[\rho]=-i \rho i_{2}$.

The Dirac operator for a free massive particle of spin $1 / 2$ is

$$
\mathbb{D}[\Phi]:=\left(\gamma_{0} \partial_{t}-\sum_{k=1}^{3} \gamma_{k} \partial_{k}+i m\right)[\Phi],
$$


where $m \in \mathbb{R}$ and $\Phi$ is a $\mathbb{C}^{4}$-valued function. Consider the quaternionic differential operator

$$
R:=P_{1}^{+}\left(i \partial_{t}+D\right)+P_{1}^{-}\left(-i \partial_{t}+D\right)-m M^{i_{2}},
$$

where $P_{k}^{ \pm}:=\frac{1}{2} M^{\left(1 \pm i i_{k}\right)}$ and $M^{\lambda}[f]:=f \lambda, \lambda \in \mathbb{H}(\mathbb{C})$. Using the algebraic properties of $A$ and $A^{-1}$ we obtain the following equality $\llbracket$ ]:

$$
R=-A \gamma_{1} \gamma_{2} \gamma_{3} \mathbb{D} A^{-1} .
$$

We consider a time-harmonic null-solution $\Phi$ of the operator $\mathbb{D}$ which has the form $\Phi(t, x)=q(x) e^{i \omega t}$, where $\omega \in \mathbb{R}$ and $q(x)$ is a $\mathbb{C}^{4}$-valued function depending only on $x$. We have the following equation for $q$

$$
\mathbb{D}_{\omega, m} q(x)=0,
$$

where $\mathbb{D}_{\omega, m}:=i \omega \gamma_{0}-\sum_{k=1}^{3} \gamma_{k} \partial_{k}+i m I$. The corresponding quaternionic reformulation of $\mathbb{D}_{\omega, m}$ is the operator

$$
D_{\alpha}:=D+M^{\alpha},
$$

where $\alpha:=-\left(i \omega i_{1}+m i_{2}\right)$. That is

$$
D_{\alpha}=-A \gamma_{1} \gamma_{2} \gamma_{3} \mathbb{D}_{\omega, m} A^{-1} .
$$

\section{Radiation condition for the Dirac operator}

In order to prove the Cauchy integral formula for null-solutions of the operator $D_{\nu}$ for the exterior domain $\mathbb{R}^{3} \backslash \bar{\Omega}$ we need some appropriate radiation condition at infinity.

Let us consider the equation

$$
D_{\nu} \mathcal{K}(x)=\delta(x), \quad \nu \neq 0, \quad x \in \mathbb{R}^{3} .
$$

When $\operatorname{Im} \nu=0$ it admits two solutions decreasing at infinity obtained (according to (7)) by the application of the operator $-D_{-\nu}$ to the fundamental solutions of the Helmholtz operator $u^{ \pm}(x):=-\frac{e^{ \pm i \nu|x|}}{4 \pi|x|}$ respectively:

$$
\mathcal{K}^{ \pm}(x)=\left(\nu+\frac{x}{|x|^{2}} \mp i \nu \frac{x}{|x|}\right) \cdot u^{ \pm}(x) .
$$

In order to omit one of these possibilities we impose the following radiation condition

$$
\left(\nu-\frac{x}{|x|^{2}}+i \nu \frac{x}{|x|}\right) \cdot \mathcal{K}(x)=o\left(\frac{1}{|x|}\right), \text { when } \quad|x| \rightarrow \infty .
$$


It is easy to see that

$$
\left(\nu-\frac{x}{|x|^{2}}+i \nu \frac{x}{|x|}\right) \mathcal{K}^{+}(x)=O\left(\frac{1}{|x|^{2}}\right), \quad \text { when }|x| \rightarrow \infty
$$

and hence $\mathcal{K}^{+}$fulfills $(14)$. For the function $\mathcal{K}^{-}$this is not true. Note that $\mathcal{K}^{+}$ is precisely the fundamental solution $\mathcal{K}_{\nu}$.

We are now ready to prove the following

Theorem 4 (Quaternionic Cauchy integral formula for the exterior domain) Let

$$
f \in C^{1}\left(\mathbb{R}^{3} \backslash \bar{\Omega} ; \mathbb{H}(\mathbb{C})\right) \cap C\left(\mathbb{R}^{3} \backslash \Omega ; \mathbb{H}(\mathbb{C})\right), \quad f \in \operatorname{ker} D_{\nu}\left(\mathbb{R}^{3} \backslash \bar{\Omega}\right), \quad \operatorname{Im} \nu \geq 0
$$

and let $f$ satisfy the radiation condition

$$
\left(\nu-\frac{x}{|x|^{2}}+i \nu \frac{x}{|x|}\right) \cdot f(x)=o\left(\frac{1}{|x|}\right), \text { when } \quad|x| \rightarrow \infty .
$$

Then

$$
f(x)=-K_{\nu}[f](x), \quad \forall x \in \mathbb{R}^{3} \backslash \bar{\Omega} .
$$

Proof. Let $\Gamma^{R}$ be a sphere with center at the origin and radius $R$ sufficiently large so that $\Omega$ is contained in the ball $B^{R}$ with boundary $\partial B^{R}=\Gamma^{R}$ (Fig. B).

According to Theorem 33 in each point $x$ of the domain $\Omega^{R}:=B^{R} \backslash \bar{\Omega}$ we have the equality

$$
f(x)=\int_{\Gamma} \mathcal{K}_{\nu}(x-y) \vec{n}(y) f(y) d \Gamma_{y}-\int_{\Gamma^{R}} \mathcal{K}_{\nu}(x-y) \frac{y}{|y|} f(y) d \Gamma_{y}^{R} .
$$


We now consider the limit of this equality when $R \rightarrow \infty$. We have the following asymptotic relation

$$
\int_{\Gamma^{R}} \mathcal{K}_{\nu}(x-y) \frac{y}{|y|} f(y) d \Gamma_{y}^{R} \sim \int_{\Gamma^{R}} \theta(y)\left(\nu-\frac{y}{|y|^{2}}+i \nu \frac{y}{|y|}\right) f(y) d \Gamma_{y}^{R}, \quad R \rightarrow \infty .
$$

Using the radiation condition we obtain that this integral tends to zero when $R \rightarrow \infty$. Thus,

$$
f(x)=\int_{\Gamma} \mathcal{K}_{\nu}(x-y) \vec{n}(y) f(y) d \Gamma_{y} .
$$

The introduction of the radiation condition in the form (14) allowed us to obtain a very simple proof of the Cauchy integral formula for the exterior domain. Nevertheless, as was shown in [7] this condition can be represented in a more elegant form. We note that the expression which appears in (14) can be rewritten as follows

$$
\left(\nu-\frac{x}{|x|^{2}}+i \nu \frac{x}{|x|}\right)=\nu\left(1+\frac{i x}{|x|}\right)+O\left(\frac{1}{|x|}\right), \quad|x| \rightarrow \infty .
$$

Thus, a natural idea is to introduce the radiation condition as

$$
\left(1+\frac{i x}{|x|}\right) f(x)=o\left(\frac{1}{|x|}\right),
$$

because the term $x /|x|^{2}$ apparently gives a faster decay. The problem here is that (16) does not imply the decay of the product $\frac{x}{|x|^{2}} \cdot f(x)$ when $|x| \rightarrow \infty$ due to the fact that $\left(1+\frac{i x}{|x|}\right)$ is a zero divisor. The following example makes this clear. Consider $f(x):=\left(1-\frac{i x}{x}\right) \cdot|x|^{2}$. This function obviously satisfies (16) but $\frac{x}{|x|^{2}} \cdot f(x)=O(|x|),|x| \rightarrow \infty$. Thus, in order to be able to apply (16) instead of (14), we should prove that if $f$ belongs to $\operatorname{ker} D_{\nu}\left(\mathbb{R}^{3} \backslash \bar{\Omega}\right)$ and satisfies (16) then it decreases at infinity. Following arguments from [7] we prove this.

Theorem 5 Let $f \in C^{1}\left(\mathbb{R}^{3} \backslash \bar{\Omega} ; \mathbb{H}(\mathbb{C})\right) \cap C\left(\mathbb{R}^{3} \backslash \Omega ; \mathbb{H}(\mathbb{C})\right), \quad f \in \operatorname{ker} D_{\nu}\left(\mathbb{R}^{3} \backslash\right.$ $\bar{\Omega}), \quad \operatorname{Im} \nu \geq 0$, such that satisfies (10). Then

$$
\int_{|x|=R}|f(x)|_{c}^{2} d \Gamma_{x}^{R}=O(1) \quad \text { as } \quad R \rightarrow \infty .
$$

Proof. Consider the expression

$$
\begin{aligned}
\left|\left(1+\frac{i x}{|x|}\right) f\right|_{c}^{2} & =\operatorname{Sc}\left(\left(1+\frac{i x}{|x|}\right) f \bar{f}^{*}\left(1+\frac{i x}{|x|}\right)\right)=2 \operatorname{Sc}\left(\left(1+\frac{i x}{|x|}\right) f \bar{f}^{*}\right)= \\
& =2\left(|f|_{c}^{2}-\operatorname{Im} \operatorname{Sc}\left(\bar{f}^{*} \frac{x}{|x|} f\right)\right) .
\end{aligned}
$$


Consequently,

$$
\begin{aligned}
\int_{|x|=R}\left|\left(1+\frac{i x}{|x|}\right) f(x)\right|_{c}^{2} d \Gamma_{x}^{R} & =2\left(\int_{|x|=R}|f(x)|_{c}^{2} d \Gamma_{x}^{R}-\right. \\
& \left.\operatorname{ImSc}\left(\int_{|x|=R} \bar{f}^{*}(x) \frac{x}{|x|} f(x) d \Gamma_{x}^{R}\right)\right) .
\end{aligned}
$$

Due to Theorem 2 we have (using the notation of Theorem 4 )

$$
\begin{aligned}
\int_{|x|=R} \bar{f}^{*}(x) \frac{x}{|x|} f(x) d \Gamma_{x}^{R} & =\int_{\Gamma} \bar{f}^{*}(x) \vec{n}(x) f(x) d \Gamma_{x}+ \\
& \int_{\Omega^{R}}\left(D_{r}\left(\bar{f}^{*}(x)\right) \cdot f(x)+\bar{f}^{*}(x) \cdot D f(x)\right) d x .
\end{aligned}
$$

The first integral on the right-hand side is some constant $C$. In order to simplify the second we use $D f=-\nu f$ and observe that

$$
D_{r} \bar{f}^{*}=-\overline{D f^{*}}=\overline{\nu^{*} f^{*}}=\nu^{*} \bar{f}^{*} .
$$

Then

$$
\begin{aligned}
\int_{\Omega^{R}}\left(D_{r}\left(\bar{f}^{*}(x)\right) \cdot f(x)+\bar{f}^{*}(x) \cdot D f(x)\right) d x & =\int_{\Omega^{R}}\left(\nu^{*} \bar{f}^{*}(x) \cdot f(x)-\nu \bar{f}^{*}(x) \cdot f(x)\right) d x= \\
& =-2 i \operatorname{Im} \nu \int_{\Omega^{R}} \bar{f}^{*}(x) \cdot f(x) d x,
\end{aligned}
$$

we obtain that

$$
\operatorname{Sc}\left(\int_{|x|=R} \bar{f}^{*}(x) \frac{x}{|x|} f(x) d \Gamma_{x}^{R}\right)=C_{0}-2 i \operatorname{Im} \nu \int_{\Omega^{R}}|f(x)|_{c}^{2} d x .
$$

Substituting this expression in (18) and using the radiation condition 116) we have

$$
\int_{|x|=R}|f(x)|_{c}^{2} d \Gamma_{x}^{R}=\operatorname{Im} C_{0}-2 \operatorname{Im} \nu \int_{\Omega^{R}}|f(x)|_{c}^{2} d x, \quad R \rightarrow \infty .
$$

The last term above vanishes when $\operatorname{Im} \nu=0$ and is negative when $\operatorname{Im} \nu>0$. $\operatorname{In}$ both cases (17) is proved.

This theorem establishes the equivalence of the radiation conditions (14) and (16) for the functions from $\operatorname{ker} D_{\nu}$.

We now obtain the radiation condition for the Dirac operator in quaternionic form, that is for $D_{\alpha}$, and prove the Cauchy integral formula for the exterior 
domain for this operator. After that, using the transform $A$ we will rewrite these results for the operator $\mathbb{D}_{\omega, m}$.

Let $\alpha$ be a purely vectorial complex quaternion $\alpha=\vec{\alpha}, \alpha \notin \mathfrak{S}, \vec{\alpha}^{2} \neq 0$ and consider $\nu:=\sqrt{\vec{\alpha}^{2}} \in \mathbb{C}$ where the sign of the root is chosen in a way such that $\operatorname{Im} \nu \geq 0$.

Let

$$
K_{\vec{\alpha}}[f](x):=P^{+} K_{\nu}[f](x)+P^{-} K_{-\nu}[f](x)
$$

where the operators $K_{ \pm \nu}$ are defined by (10) and $P^{ \pm}:=\frac{1}{2 \nu} M^{(\nu \pm \vec{\alpha})}$ are two mutually complementary projection operators in the set of $\mathbb{H}(\mathbb{C})$-valued functions.

In the case when $\alpha:=\vec{\alpha} \in \mathfrak{S}, \nu=0$, we introduce the operator $K_{\vec{\alpha}}$ as follows

$$
K_{\vec{\alpha}} f:=K_{0} f-M^{\vec{\alpha}} V_{0} f
$$

where

$$
V_{0}[f](x):=\int_{\Gamma} \theta_{0}(x-y) \vec{n}(y) f(y) d \Gamma_{y}, \quad x \in \mathbb{R}^{3} \backslash \Gamma .
$$

Here $\theta_{0}(x-y)=-\frac{1}{4 \pi|x-y|}$.

For all $\alpha \in \mathbb{H}(\mathbb{C})$ (that is $\alpha \in \mathfrak{S}$ or $\alpha \notin \mathfrak{S}$ ), $K_{\vec{\alpha}} f$ admits the following representation

$$
\begin{array}{r}
K_{\vec{\alpha}}[f](x)=-\int_{\Gamma}\left\{\theta_{\nu}(x-y)\left(\frac{x-y}{|x-y|^{2}}-\frac{i \nu(x-y)}{|x-y|}\right) \vec{n}(y) f(y)+\right. \\
\left.\quad+\theta_{\nu}(x-y) \vec{n}(y) f(y) \vec{\alpha}\right\} d \Gamma_{y}, \quad x \in \Gamma .
\end{array}
$$

Theorem 6 Let $f \in C^{1}\left(\mathbb{R}^{3} \backslash \bar{\Omega} ; \mathbb{H}(\mathbb{C})\right) \cap C\left(\mathbb{R}^{3} \backslash \Omega ; \mathbb{H}(\mathbb{C})\right), f \in \operatorname{ker} D_{\alpha}\left(\mathbb{R}^{3} \backslash \bar{\Omega}\right)$, $\alpha \in \mathbb{H}(\mathbb{C})$, Sc $\alpha=0$ such that $f$ satisfies the radiation condition

$$
\nu f(x)+\frac{i x}{|x|} f(x) \vec{\alpha}=o\left(\frac{1}{|x|}\right), \quad \text { when }|x| \rightarrow \infty,
$$

where $\nu:=\sqrt{\bar{\alpha}^{2}} \in \mathbb{C}$ and $\operatorname{Im} \nu \geq 0$. If $\alpha \in \mathfrak{S}$ then we suppose additionally that $f(x)=o(1)$. Then

$$
f(x)=K_{\vec{\alpha}}[f](x), \quad \forall x \in \mathbb{R}^{3} \backslash \bar{\Omega} .
$$


Proof. First we suppose that $\vec{\alpha} \notin \mathfrak{S}$. Multiplying (22) by $\vec{\alpha} / \nu$ from the right-hand side we obtain

$$
f(x) \vec{\alpha}+\nu \frac{i x}{|x|} f(x)=o\left(\frac{1}{|x|}\right)
$$

adding and subtracting (22) and (23) we obtain

$$
\begin{gathered}
\left(\nu f(x)+\frac{i x}{|x|} f(x) \vec{\alpha}\right) \pm\left(f(x) \vec{\alpha}+\nu \frac{i x}{|x|} f(x)\right)= \\
=\left(1 \pm \frac{i x}{|x|}\right) f(x) \nu \pm\left(1 \pm \frac{i x}{|x|}\right) f(x) \vec{\alpha}= \\
=\left(1 \pm \frac{i x}{|x|}\right) f(x)(\nu \pm \vec{\alpha})=o\left(\frac{1}{|x|}\right) .
\end{gathered}
$$

Which can be writen as follows

$$
P^{+}\left(\left(1+\frac{i x}{|x|}\right) f(x)\right)+P^{-}\left(\left(1-\frac{i x}{|x|}\right) f(x)\right)=o\left(\frac{1}{|x|}\right) \quad \text { when }|x| \rightarrow \infty \text {. }
$$

Thus, (22) is equivalent to (25), from which it can be seen that $P^{+} f$ fulfills the radiation condition (16) and $P^{-} f$ fulfills its conjugate which corresponds to the operator $D_{-\nu}$. Consequently the integrals $K_{ \pm \nu} P^{ \pm} f$ taken over the sphere $\Gamma^{R}$ (see the proof of Theorem [) decrease when $R \rightarrow \infty$.

Since $K_{ \pm \nu}$ commute with $P^{ \pm}$we obtain that the integral $K_{\vec{\alpha}} f(x)$ taken over the sphere $\Gamma^{R}$ also decreases when $R \rightarrow \infty$.

In the case when $\alpha$ is a zero divisor the radiation condition (22) becomes

$$
\frac{i x}{|x|} f \vec{\alpha}=o\left(\frac{1}{|x|}\right), \quad \text { when }|x| \rightarrow \infty .
$$

Since the behavior of $\theta_{0}$ in (21) is of the type $O\left(\frac{1}{|x|}\right)$ when $|x| \rightarrow \infty$ and since the expression $M^{\vec{\alpha}} V_{0}$ contains the multiplication from the right-hand side by $\vec{\alpha}$, and in this case $f \vec{\alpha}=o\left(\frac{1}{|x|}\right)$, it can be seen that the integral (20) taken over the sphere $\Gamma^{R}$ decreases at infinity also.

We now consider the Dirac operator $D_{\vec{\alpha}}, \vec{\alpha}:=-\left(i \omega i_{1}+m i_{2}\right)$. Note that $\vec{\alpha} \in \mathfrak{S}$ iff $\omega^{2}=m^{2}$. In the following lines we will obtain the radiation condition for the Dirac operator in its classical representation using $\gamma$-matrices.

Let us denote

$$
q:=A^{-1} f
$$


According to (13), if $f \in \operatorname{ker} D_{\vec{\alpha}}$ then $q \in \operatorname{ker} \mathbb{D}_{\omega, m}$. Suppose that $f$ fulfills the radiation condition (22) then we have

$$
\nu A \gamma_{1} \gamma_{2} \gamma_{3} A^{-1} f+A \gamma_{1} \gamma_{2} \gamma_{3} A^{-1} \frac{i x}{|x|} f \vec{\alpha}=o\left(\frac{1}{|x|}\right) .
$$

Let us denote

$$
f \vec{\alpha}=: F
$$

and consider the product

$$
x F=\left(x_{1} i_{1}+x_{2} i_{2}+x_{3} i_{3}\right) F .
$$

Using properties 1.-3. of the transforms $A$ and $A^{-1}$ (Section 2.3), we obtain

$$
x F=A \gamma_{1} \gamma_{2} \gamma_{3}\left(\sum_{k=1}^{3} x_{k} \gamma_{k}\right) A^{-1}[F] .
$$

Consequently, using the properties 4 . and 5., we have

$$
\begin{gathered}
x F(x)=A \gamma_{1} \gamma_{2} \gamma_{3}\left(\sum_{k=1}^{3} x_{k} \gamma_{k}\right) A^{-1}\left[f(x) \cdot\left(-i \omega i_{1}-m i_{2}\right)\right]= \\
=A \gamma_{1} \gamma_{2} \gamma_{3}\left(\sum_{k=1}^{3} x_{k} \gamma_{k}\right) A^{-1} A \gamma_{1} \gamma_{2} \gamma_{3} \gamma_{0} A^{-1} f(x) \cdot(-i \omega)+ \\
+A \gamma_{1} \gamma_{2} \gamma_{3}\left(\sum_{k=1}^{3} x_{k} \gamma_{k}\right) A^{-1} A \gamma_{1} \gamma_{2} \gamma_{3} A^{-1} f(x) \cdot(-i m)= \\
=A\left(\sum_{k=1}^{3} x_{k} \gamma_{k}\right) \gamma_{0} A^{-1} f(x) \cdot(-i \omega)+A\left(\sum_{k=1}^{3} x_{k} \gamma_{k}\right) A^{-1} f(x) \cdot(-i m) .
\end{gathered}
$$

Using (27) we can rewrite the previous equation as

$$
x F=A\left(\sum_{k=1}^{3} x_{k} \gamma_{k}\right)\left(-i \omega \gamma_{0}-i m\right) q .
$$

Thus (22) can be rewritten as follows

$$
\nu f+\frac{i x}{|x|} f \vec{\alpha}=\nu A q+A \frac{1}{|x|} \sum_{k=1}^{3} x_{k} \gamma_{k}\left(\omega \gamma_{0}+m\right) q=o\left(\frac{1}{|x|}\right),
$$


and hence the radiation condition for the Dirac operator in its traditional form is obtained in the following form

$$
\nu q(x)-\left(\omega \gamma_{0}-m\right) \frac{\overrightarrow{x_{\gamma}}}{|x|} q(x)=o\left(\frac{1}{|x|}\right), \quad|x| \rightarrow \infty
$$

where $\overrightarrow{x_{\gamma}}:=\sum_{k=1}^{3} x_{k} \gamma_{k}$.

We introduce the following operator

$$
\mathbb{K}_{\omega, m}:=A^{-1} K_{\vec{\alpha}} A,
$$

where $\vec{\alpha}=-\left(i \omega i_{1}+m i_{2}\right)$. Using this operator, in [4] an analogue of the Cauchy integral formula for the Dirac operator $\mathbb{D}_{\omega, m}$ in bounded domains was proved. Here with the aid of the obtained radiation condition we obtain this fact for the unbounded domains.

Theorem 7 (Cauchy integral formula for $\mathbb{D}_{\omega, m}$ in the exterior domain $\mathbb{R}^{3} \backslash \bar{\Omega}$ ) Let $q \in C^{1}\left(\mathbb{R}^{3} \backslash \bar{\Omega} ; \mathbb{H}(\mathbb{C})\right) \cap C\left(\mathbb{R}^{3} \backslash \Omega ; \mathbb{H}(\mathbb{C})\right), q \in \operatorname{ker} \mathbb{D}_{\omega, m}\left(\mathbb{R}^{3} \backslash \bar{\Omega}\right)$, and $q$ satisfies the radiation condition (28). Then

$$
q(x)=\mathbb{K}_{\omega, m}[q](x), \quad x \in\left(\mathbb{R}^{3} \backslash \bar{\Omega}\right) .
$$

The proof consists in the application of 13 ) and Theorem 4 .

Acknowledgment: This work was partially supported by CONACYT, project 32424-E.

\section{References}

[1] Bogoliubov N. N.and Shirkov D. V. Quantum fields. Moscow: Fizmatlit, 1993, 336 pp. (in Russian).

[2] Gürlebeck K. Hypercomplex factorization of the Helmholtz equation. Zeitschrift für Analysis und ihre Anwendungen, 1986, v. 5, \#2, 125-131.

[3] Gürlebeck K. and Sprössig W. Quaternionic and Clifford calculus for physicists and engineers. John Wiley \& Sons, 1997.

[4] Kravchenko V. V. On a biquaternionic bag model. Zeitschrift für Analysis und ihre Anwendungen,1995, v. 14, \#1, 3-14.

[5] Kravchenko V. V. On the relation between holomorphic biquaternionic functions and time-harmonic electromagnetic fields. Deposited in UkrINTEI, 29.12.1992, \#2073-Uk-92,18pp. (in Russian).

[6] Kravchenko V. V. and Shapiro M. V. Integral representations for spatial models of mathematical physics. Addison Wesley Longman Ltd., Pitman Res., Notes in Math Series, v. 351, 1996. 
[7] McIntosh A. and Mitrea M. Clifford algebras and Maxwell's equations in Lipschitz domains. Mathematical Methods in the Applied Sciences, 1999, v. $22,1599-1620$.

[8] Müller C. Grund probleme der mathematischen Theorie elektromagnetischer Schwingungen. Springer-Verlag, Berlin, 1957.

[9] Silver S. Microwave antenna theory and design. M.I.T. Radiation Laboratory Series. Vol. 12, Mc Graw-Hill, New York, 1949.

[10] Sommerfeld A. Die Greensche Funktion der Schwingungsgleichung. Jber. Deutsch. Math. Verein. 21, 309-353, 1912.

[11] Thaller B. The Dirac equation. Springer-Verlag, 1992.

[12] Vladimirov V. S. Equations of mathematical physics. Moscow: Nauka, 1984 (in Russian); Engl. transl. of the first edition: N.Y.: Marcel Dekker, 1971. 
This figure "Fig.jpg" is available in "jpg" format from: http://arxiv.org/ps/math-ph/0008042v1 